\title{
The Mg II index for upper atmosphere modelling
}

\author{
G. Thuillier and S. Bruinsma \\ Service d'Aéronomie, BP 3, 91371 Verrières-le-Buisson, France \\ Received: 19 April 2000 - Revised: 27 October 2000 - Accepted: 12 December 2000
}

\begin{abstract}
The solar radio flux at $10.7 \mathrm{~cm}$ has been used in upper atmosphere density modelling because of its correlation with EUV radiation and its long and complete observational record. A proxy, the Mg II index, for the solar chromospheric activity has been derived by Heath and Schlesinger (1986) from Nimbus-7 data. This index allows one to describe the changes occurring in solar-activity in the UV Sun spectral irradiance. The use of this new proxy in upper atmosphere density modelling will be considered. First, this is supported by the $99.9 \%$ correlation between the solar radio flux (F10.7) and the Mg II index over a period of 19 years with, however, large differences on time scales of days to months. Secondly, correlation between EUV emissions and the Mg II index has been shown recently, suggesting that this last index may also be used to describe the EUV variations. Using the same density dataset, a model was first run with the F10.7 index as a solar forcing function and second, with the Mg II index. Comparison of their respective predictions to partial density data showed a 3-8\% higher precision when the modelling uses the Mg II index rather than F10.7. An external validation, by means of orbit computation, resulted in a $20-40 \%$ smaller RMS of the tracking residuals. A density dataset spanning an entire solar cycle, together with $\mathrm{Mg}$ II data, is required to construct an accurate, unbiased as possible density model.
\end{abstract}

Key words. Atmospheric composition and structure (middle atmosphere - composition and chemistry; thermosphere - composition and chemistry) - History of geophysics (atmospheric sciences)

\section{Introduction}

Thermosphere models, representing temperature and (partial) density as a function of environmental parameters, are used in upper atmosphere studies, and also in satellite orbit

Correspondence to: G. Thuillier

(gerard.thuillier@aerov.jussieu.fr) determination to compute the atmospheric drag force. Precise satellite orbit computation, for Earth observation as in case of oceanographic satellites, also requires the estimation of many density scale factors to absorb the errors in orbit predictions induced by the density model. This is only possible when a tracking system is available, with stringent requirements concerning the accuracy, and temporal and spatial continuity of its observations. A more accurate drag estimate and consequently, a more realistic density model than what is available nowadays, is required to relax this operational constraint. The reasons for the slow progress in density modelling since the models as early as J71 (Jacchia, 1971) are the following, though not necessarily in this order:

1. the simplified description of atmospheric phenomena;

2. the use of proxy indicators for solar and geomagnetic atmospheric heating processes;

3. the sparse partial and total density, and temperature data available with their own precision and accuracy.

The first item of the list is the most difficult subject to tackle; this is why recent empirical density models such as DTM94 (Berger et al., 1998) or MSIS86 (Hedin et al., 1987) are still based upon the same algorithms as their earliest versions. The basic difference between the first DTM model, DTM78 (Barlier et al., 1978), and DTM94, is the quantity and quality of density data incorporated in the model, allowing the modelling of smaller density variations. However, large regions of the upper atmosphere remain sparsely observed, notably in the $100-200 \mathrm{~km}$ altitude range.

This study is concerned by the heating of the atmosphere by solar extreme ultraviolet (EUV) radiation. As our database of temperature, and partial and total density extends over several years, it is necessary to dispose of a similar EUV database for the purpose of modelling. This is not achieved. Furthermore, the EUV being totally absorbed by the upper atmosphere, is only observable from space, where instruments may suffer from ageing, with obvious consequences concerning its absolute irradiance. This is why a proxy of 
EUV radiation is used. The F10.7 radio emission has been measured on a daily basis by the National Research Council of Canada since 1947 and constitutes a complete record. As the F10.7 radio emission originates in the high chromosphere/lower solar corona, it is frequently used to model the heating of the upper atmosphere by the solar extreme ultraviolet radiation.

The EUV radiation originates in the solar high chromosphere, the transition region and the corona, including emission lines at different temperatures, while the radio flux at $10.7 \mathrm{~cm}$ originates at a specific altitude of the solar atmosphere. This is the reason why looking for an EUV variability proxy is not a straightforward task. By calculating averaged values over 3 solar rotations, the mean radio flux is correlated with the mean EUV radiation (up to 0.99). But the correlation is weaker between the daily values (approximately 0.88 ). Correlations between EUV and density are 0.97 and 0.85 for mean and daily values, respectively (Hedin, 1984). However, F10.7 suffers from several defects as an EUV proxy due to: the temperature dependence of the various emissions composing this domain, their specific process of emission, and the optical depth of the solar atmosphere at the wavelength of emission (Neupert, 1998). Furthermore, the EUV emission exhibits a 13-day periodicity which is not present in the F10.7 time series (Donnelly and Puga, 1990). Consequently, F10.7 cannot represent all the EUV emissions accurately, especially on a short term basis.

As the EUV lines variability is linked to the structure of the solar magnetic field, the extent and brightness of the Ca II plages have been considered as a UV domain proxy (Lean et al., 1982) for the 145-200 nm domain. Below that, Neupert (1998) correlated the Fe IX to Fe XVI emission lines with the Ca II plages area and brightness, and suggested to use the Ca II index to describe the EUV variability (White et al., 1992). In fact, such an attempt was made by Hedin (1984) by correlating thermospheric densities with $\mathrm{Ca}$ II index and F10.7. Correlations with density appear better with F10.7 than with the Ca II index, especially on a long term basis. However, significant differences were pointed out on a short term basis when using F10.7. Hedin (1984) also concluded that regular measurements are required to make the $\mathrm{Ca}$ II data useful. Even today the Ca II database suffers from missing measurements. The largest datatset spans from 1942 to 1987 with an average usable coverage from 1980 to 1983 (epoch of Dynamic Explorer 2 density data) of 50\%, which is too low for our modelling purpose. This is the reason why another proxy is needed.

First, the Mg II index exhibits a 13-day periodicity (Cebula and DeLand, 1998) as the EUV emissions. Second, Avrett (1992) showed, from a theoretical study, that Mg II and $\mathrm{Ca}$ II emission lines originate at nearly the same altitude in the high chromosphere. Consequently, they should behave similarly. Indeed, both indices are correlated (0.975), as shown by Donnelly et al. (1994) and as expected, the Mg II index succeeds in describing the UV emission variability down to the Ly $\alpha$ emission (121.6 nm), as shown by Lean et al. (1992). Below Ly $\alpha$ little information is available. A

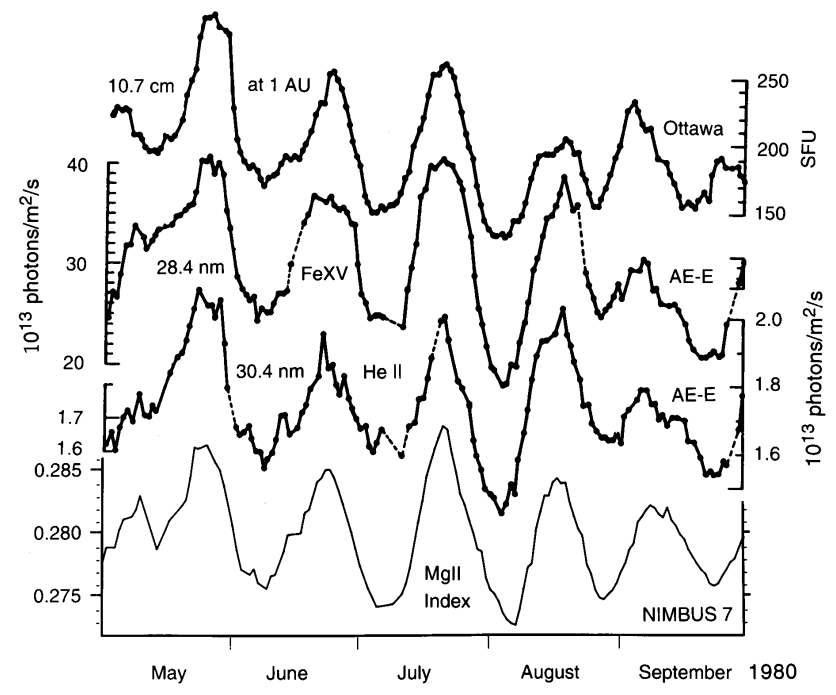

Fig. 1. The Mg II index (bottom curve), the $30.4 \mathrm{~nm}$ (He II) and $28.4 \mathrm{~nm}(\mathrm{Fe} \mathrm{XV}$ ) from AE-E, and the $10.7 \mathrm{~cm}$ solar emissions from May to September 1980 (top plot extracted from Avrett, 1992).

recent correlation has been shown between the helium line at $30.4 \mathrm{~nm}$ (He II) measured on board SOHO, and the Mg II variation observed at the same time (Viereck and Judge, personal communication), suggesting to use the $\mathrm{Mg}$ II index to describe the EUV heating of the thermosphere. Fig. 1 shows the evolution of F10.7, the Fe XV emission at $28.4 \mathrm{~nm}$, the He II emission at $30.4 \mathrm{~nm}$ (extracted from Fig. 1 of Avrett, 1992), as well as that of the Mg II index from 1 May 1980 to 1 October 1980. During the first two weeks in May, as well as at the end of September, F10.7 is out of phase with respect to the EUV emissions (it is declining instead of rising). Furthermore, the EUV maximum in August and September is a high one followed by a smaller one, while F10.7 exhibits the opposite evolution. Consequently, the correlation of $\mathrm{Mg}$ II with Fe XV and He II is higher than that of F10.7. The complexity of the matter is revealed by Fig. 1 as well, which shows important differences between the four observables.

The NOAA-9 spacecraft carrying the SBUV-2 spectrometer failed in February 1998 after 12 years of operations. Furthermore, observations were degraded by the end of the mission. The SOLSTICE data onboard UARS have been used to complement the SBUV measurements by Viereck and Puga (1999), who built a 20-year time series of $\mathrm{Mg}$ II indices.

Another reason for choosing Mg II versus Ca II, besides from the availability of the indices, is the presence of the noise in the data. We have compared simultaneous measurements of Mg II indices from NOAA-9 and NOAA-11, operating at the same time (see references below). RMS differences are not greater than $1 \%$. The Ca II index measured from Kitt Peak and Sacramento Peak, between 1984 and 1990, have a RMS difference of about 6\% (White et al., 1992). This may be caused by the atmospheric transparency and the greater amplitude of the $\mathrm{H}$ and $\mathrm{K}$ chromospheric components of the $\mathrm{Mg}$, rather than the Ca Fraunhofer lines (Donnelly et al., 
1994). Consequently, the density modelling should become better by using the Mg II index rather than F10.7.

The data available for upper atmosphere modelling have been obtained from different sources: satellite drag total density, in situ measurements of the atmospheric constituents with mass spectrometers, temperature profiles by satellite borne spectrometers and interferometers, and incoherent scatter radars. The measurement of atmospheric constituents with mass spectrometers provides the distribution of a gas as a function of altitude, local time, latitude, and solar and geomagnetic activity, with a convenient temporal and spatial resolution for our purpose. The problem we encountered is that there is only one large density dataset concurrent with Mg II, namely that of the Dynamics Explorer 2 (DE-2) mass spectrometer (Carignan et al., 1981), while all other datasets, in particular the Atmosphere Explorer-C, -D and -E partial density datasets (Nier et al., 1973; Spencer et al., 1973), and the total density derived from the space borne microaccelerometer CACTUS (Villain, 1980) have been obtained before the determination of the $\mathrm{Mg}$ II index, which started in 1978.

Future total density data will be provided by a microaccelerometer placed onboard the CHAMP spacecraft (Reigber et al., 1996), which was launched in July 2000. Combined with the ongoing observation of the Mg II doublet, the test model elaborated in this study will be refined using the CHAMP density dataset obtained at the same time.

The next section describes the Mg II characteristics and the DE-2 partial density data. The DTM model equations are briefly reviewed. The third section presents the results in terms of partial density residual RMS and precise orbit computation with the F10.7 and Mg II indices. The problems encountered during the study and the future plans are discussed in Sect. 4. A conclusion is presented at the end of this paper.

\section{The data}

\subsection{Mg II data}

The Mg II index was proposed by Heath and Schlesinger (1986). In the core of the Mg II Fraunhofer line, the H and K chromospheric emissions are observable. By calculating the ratio between the chromospheric emission and the solar continuum, the Mg II index is obtained. As these measurements are taken using a spectrometer having certain spectral characteristics, the $\mathrm{Mg}$ II index depends on its slit function and the way of estimating the solar continuum. We have used the Mg II definition given by Cebula and DeLand (1998). It is quasi independent of the instrument calibration and possible degradation exists due to ageing.

The index used in this study has been derived from instruments onboard satellites from the National Oceanic and Atmospheric Administration (NOAA), and has been corrected for instrumental errors (Cebula et al., 1998). The Mg II data from Nimbus-7, NOAA-9 and NOAA-11, derived from

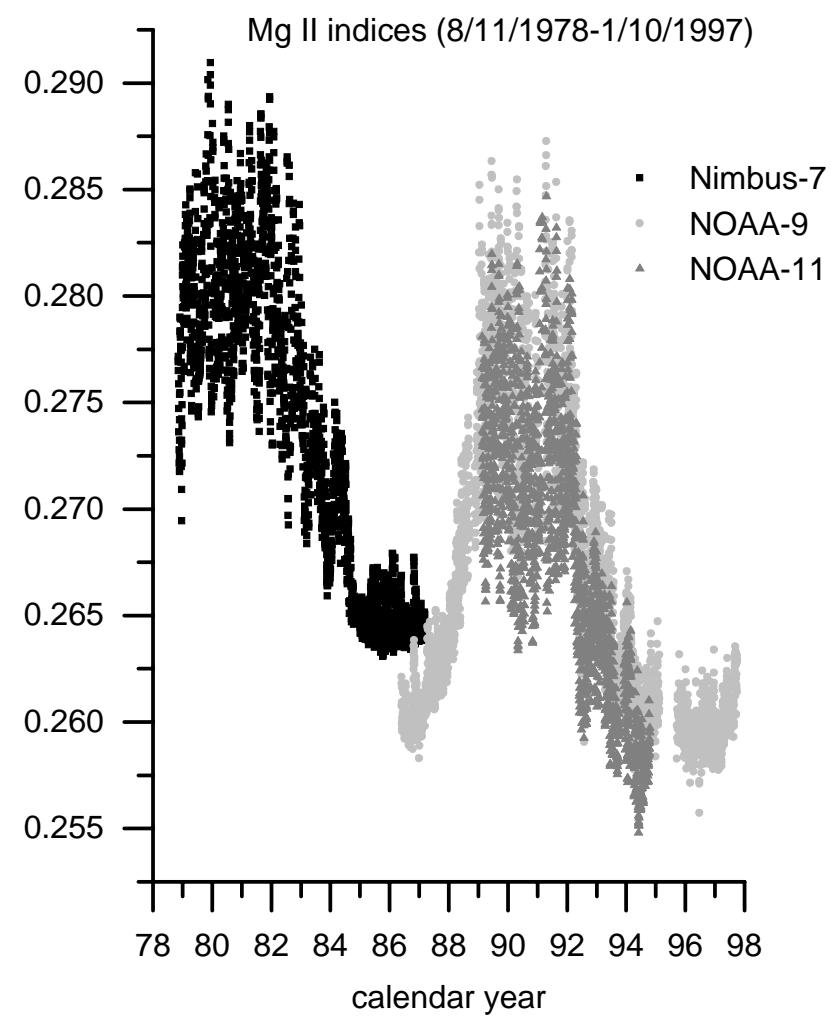

Fig. 2. The corrected for instrumental drift original Nimbus-7, NOAA-9 and NOAA-11 Mg II indices datasets.

the SBUV (Solar Backscatter Ultraviolet) onboard Nimbus7 and SBUV/2 instruments onboard the latter two satellites (DeLand and Cebula, 1993), have been made available. The first observations by Nimbus-7 took place in November 1978. Since that date the index is available 4 out of 5 days, in general, but data gaps exceeding one month exist due to instrumental or orbit control errors. Figure 2 presents the Mg II index derived from the SBUV and SBUV/2 instruments onboard the three NOAA satellites. The offsets that exist between the three datasets are due to small instrumental differences (minor differences in wavelength sampling and slit function), since two instruments are never perfectly identical. The difference between the two SBUV/2 instruments is small, but the SBUV data are approximately $4 \%$ larger than the NOAA-9 data. Thanks to overlapping observation intervals, the Nimbus-7 and NOAA-11 data have been fitted to the NOAA-9 scale by linear regression, as described by DeLand and Cebula (1993), resulting in a composite Mg II dataset.

The composite data span 19 years on a single scale, from November 1978 to October 1997, but there are still missing observations. Atmospheric density models like DTM94 require a mean solar radio flux $(\overline{\mathrm{F}} 10.7)$ averaged over the three last solar rotations. Since the DTM94 algorithms will be used, we have a similar requirement for the $\mathrm{Mg}$ II index. As gaps are existing, a cubic spline in case of one or two consecutive missing days has been used to insure continuity. When the data gap is greater than two days, a realistic interpolation 


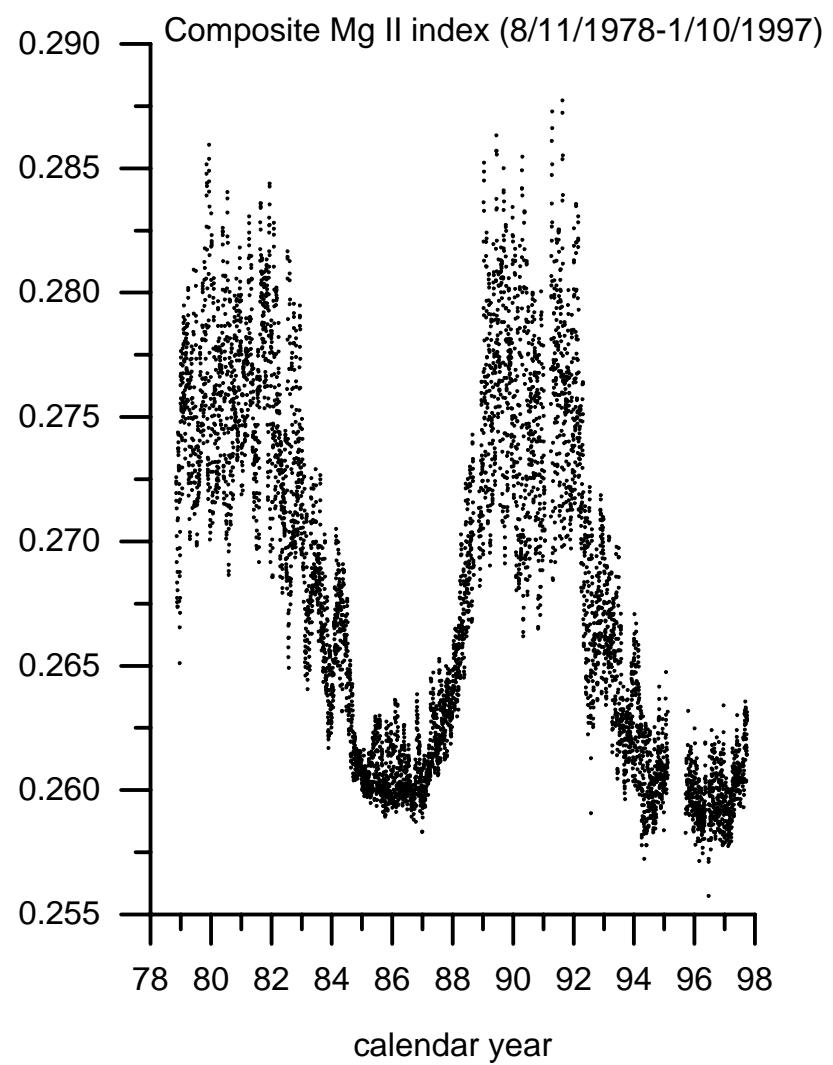

Fig. 3. The composite Mg II index scaled to the NOAA-9 observations. Data gaps of less than three days have been filled by interpolation.

is not possible and the existing gaps are left. The composite dataset after interpolation, shown in Fig. 3, exhibits a variability as expected from the Schwabe cycle.

Comparing F10 .7 and Mg II datasets shows the following:

- the trend is similar as expected from the Schwabe cycle;

- the short time variations are not identical.

Assuming a linear dependence, we obtained by a leastsquares calculation, the following relation:

$\mathrm{Mg}$ II index $=0.000128 \mathrm{~F} 10.7+0.25068$

The correlation between Mg II and F10.7 is 0.99 over the entire dataset, but the RMS difference is 25 solar flux units (sfu). Usually models require solar indices to be averaged over one or three solar rotations. Applying the same averaging to the F10.7 and Mg II index, the correlation remains 0.99 , but the scatter reduces to $10 \mathrm{sfu}$. This shows that the main difference between the $\mathrm{Mg}$ II index and F10.7 occurs at short temporal scale, as anticipated.

Figure 4 presents the rescaled composite $\mathrm{Mg}$ II index in sfu and the difference with F10.7. The largest differences occur during solar cycle maximum, associated with the solar rotation. It is known that the solar rotation generates significant variations in UV and EUV, as observed from Earth, due to

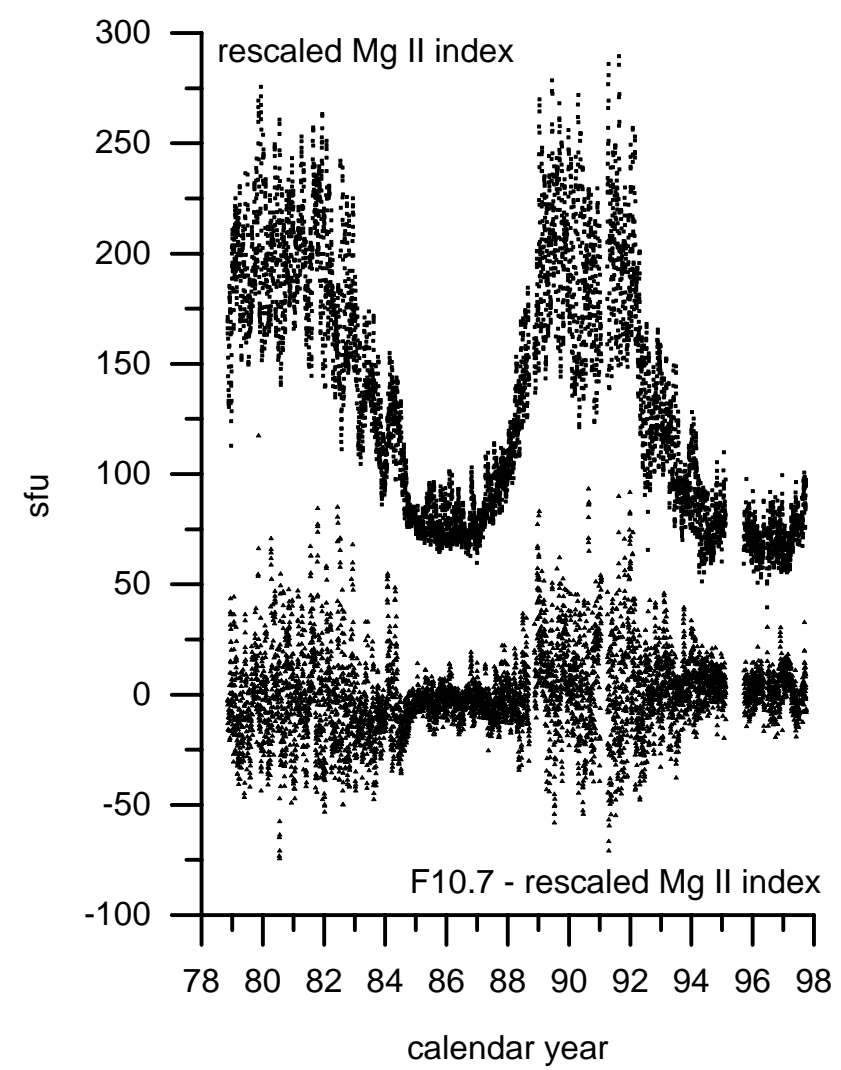

Fig. 4. The rescaled Mg II index (top) and the difference with F10.7.

the appearance of sunspots and associated phenomena. This result may be interpreted as supporting the use of the $\mathrm{Mg}$ II index for providing a better description of solar irradiance change associated with the solar activity. However, despite the data correction for instrumental drift made by Cebula et al. (1998), they still seem trended. Since the trend is smaller than $1 \mathrm{sfu} / \mathrm{year}$, and the data are being used only over a twoyear period, it has been ignored in this study.

\subsection{DE-2 partial density and temperature data}

To produce a new density model based on the initial DTM94 model, partial derivatives, with respect to density and temperature observations, have to be calculated, which are then used in a least-squares adjustment of the model coefficients. DTM94 has been elaborated using CACTUS and satellite drag total density, DE-2 (temperature and constituents) and OGO-6 temperature data (Thuillier et al., 1977), under low and high solar activity.

The DE-2 data cover the last part of the maximum and part of the declining phase of solar cycle 21, from July 1981 to January 1983. The neutral mass spectrometer (NACS, Carignan et al., 1981) onboard DE-2 measured $\mathrm{N}_{2}, \mathrm{O}, \mathrm{He}$, and $\mathrm{Ar}$ densities, while the temperature was measured by the wind and temperature spectrometer (WATS, Spencer et al., 1981). Unfortunately, NACS did not measure absolute quantities, and an instrumental calibration factor for each constituent 
had to be estimated from the data itself (Hedin, 1987). The precision is better than $5 \%$, and a sampling rate of the order of seconds provides a high temporal resolution of the measurements. The measured temperatures do not suffer from an instrumental bias (Berger et al., 1998). The spatial coverage of the DE-2 dataset is adequate with respect to our need, since the satellite was in a polar eccentric orbit, measuring from 225 to $1100 \mathrm{~km}$ altitude.

\subsection{The DTM model equations}

The partial densities of $\mathrm{O}, \mathrm{O}_{2}, \mathrm{~N}_{2}, \mathrm{He}$, and $\mathrm{H}$ are given in the DTM models at $120 \mathrm{~km}$ altitude. Using an exponential height distribution function $f_{i}$ per constituent $i$ (Barlier et al., 1978) and a Bates temperature profile (Bates, 1959), the neutral density may be calculated at higher altitudes, up to approximately $1500 \mathrm{~km}$. The total density at altitude $z$ is the sum of the partial densities:

$\rho(z)=\sum_{i} \frac{m_{i}}{N_{A}} c_{i}(120 \mathrm{~km}) f_{i} \exp \left(G_{i}(L)\right)$

where $m_{i}$ is the molecular or atomic mass, $N_{A}$ Avogadro's number, $c_{i}(120 \mathrm{~km})$ the mean partial concentration at 120 $\mathrm{km}$ and $G(L)$ the spherical harmonic expansion of density variation with the environmental parameters, represented by $L$. The environmental input parameters $L$ represent altitude, latitude, longitude, local time, geomagnetic activity $K p$, the day of the year and a mean and daily solar activity indicator. The new set of model coefficients was calculated using the rescaled Mg II rather than the original Mg II index. This allows one to use the model even when the Mg II index is not available (data gaps, or typically prior to 1978). DTM94 models the variations in atmospheric density with 38 coefficients for the exospheric temperature and each atmospheric constituent, but the temperature at $120 \mathrm{~km}$ and its gradient are represented by constant values. The function $G$ contains non-periodic terms $(N P)$ which depend on solar and geomagnetic activity and latitude. The periodic terms have annual $(P A)$, semi-annual $(P S A)$, diurnal $(P D)$, semidiurnal $(P S D)$ and terdiurnal $(P T D)$ periods for each constituent. This can be expressed as follows:

$$
\begin{gathered}
G=\sum_{i} a_{i} N P+\sum_{j} a_{j} P A+\sum_{k} a_{k} P S A+\sum_{l} a_{l} P D \\
+\sum_{m} a_{m} P S D+\sum_{n} a_{n} P T D
\end{gathered}
$$

The effects of phenomena with periods smaller than 8 hours, such as gravity waves, or nonperiodic, such as solar flares, are not represented in the model, but constitute sources of noise in the data when the terdiurnal and larger period signals are estimated.

Analysis of the DTM94 residuals shows the possibility of increasing the number of coefficients. For the present modelling, in order to better represent the exospheric temperature and the partial $\mathrm{O}, \mathrm{N}_{2}$ and He densities, 5 coefficients per component have been added, which is allowed by the large amount of observations (approximately 1.3 million), and most of the DTM94 coefficients have been re-estimated. The new coefficients have been retained if they could be estimated from the available data with a standard deviation of at least an order of magnitude smaller than the estimate, and if their contribution to the total variation is at least $0.5 \%$. These conditions apply to all estimated coefficients. The annual variations have been determined despite the short time span of the DE-2 data. The temperature at $120 \mathrm{~km}$ is, in this study, modelled as a function of latitude and mean solar activity (NP terms), as suggested by Oliver (1979).

\section{Results}

The residuals, in this paper defined as Observed/Calculated $(\mathrm{O} / \mathrm{C}$ instead of the usual $\mathrm{O}-\mathrm{C})$, reflect the precision of the models. The DE-2 mass spectrometer does not provide absolute densities; the use of its measurements require scaling by a calibration factor. These have not been derived in this study, but the scaling factors that were used in the construction of DTM94 were taken. The mean value of the residuals corresponds to the instrumental calibration factor, which is different for each constituent, and to the model capability to represent the observations. An erroneous value of a calibration factor, which in the case of DE-2 has an uncertainty of approximately $30 \%$, thus, causes a model bias. The RMS of the residuals about the mean value reflects the short-term modelling performance.

The comparison between F10.7 and Mg II model performance is achieved by using the same DE-2 data, with F10.7 and the Mg II index, resulting in two test models named DTM-F107 and DTM-Mg II, respectively. The standard deviations of the estimated coefficients are nearly identical for both test models, as is to be expected for estimation under identical conditions. Taking the limited amount of DE-2 data (under high solar activity conditions) into consideration, we have compared the test models outputs under low solar activity conditions to DTM94 to check for possible model bias in that condition. These comparisons showed differences of up to $10 \%$ under high (220 sfu) and up to $20 \%$ under low (70 sfu) solar activity conditions. This shows that the test models have acceptable performance even at low solar activity. No other comparisons were made due to the different database used by DTM94 and the test models, as well as the addition of 20 coefficients in the $G(L)$ function to the latter.

The residuals between DE- 2 data and the test models have been divided into altitude, latitude, local time, month, F10.7 - $\overline{\mathrm{F}} 10.7, \overline{\mathrm{F}} 10.7$, and $K p$ bins. The mean and RMS of the residuals have then been computed per bin, for which specific results per constituent will be shown in Figs. 5 through 8. The modelling results for the temperature and the constituents are given in the following subsections.

\subsection{The temperature modelling}

The temperature modelling in terms of both bias and precision is the best with DTM-Mg II, as may be seen in the last 

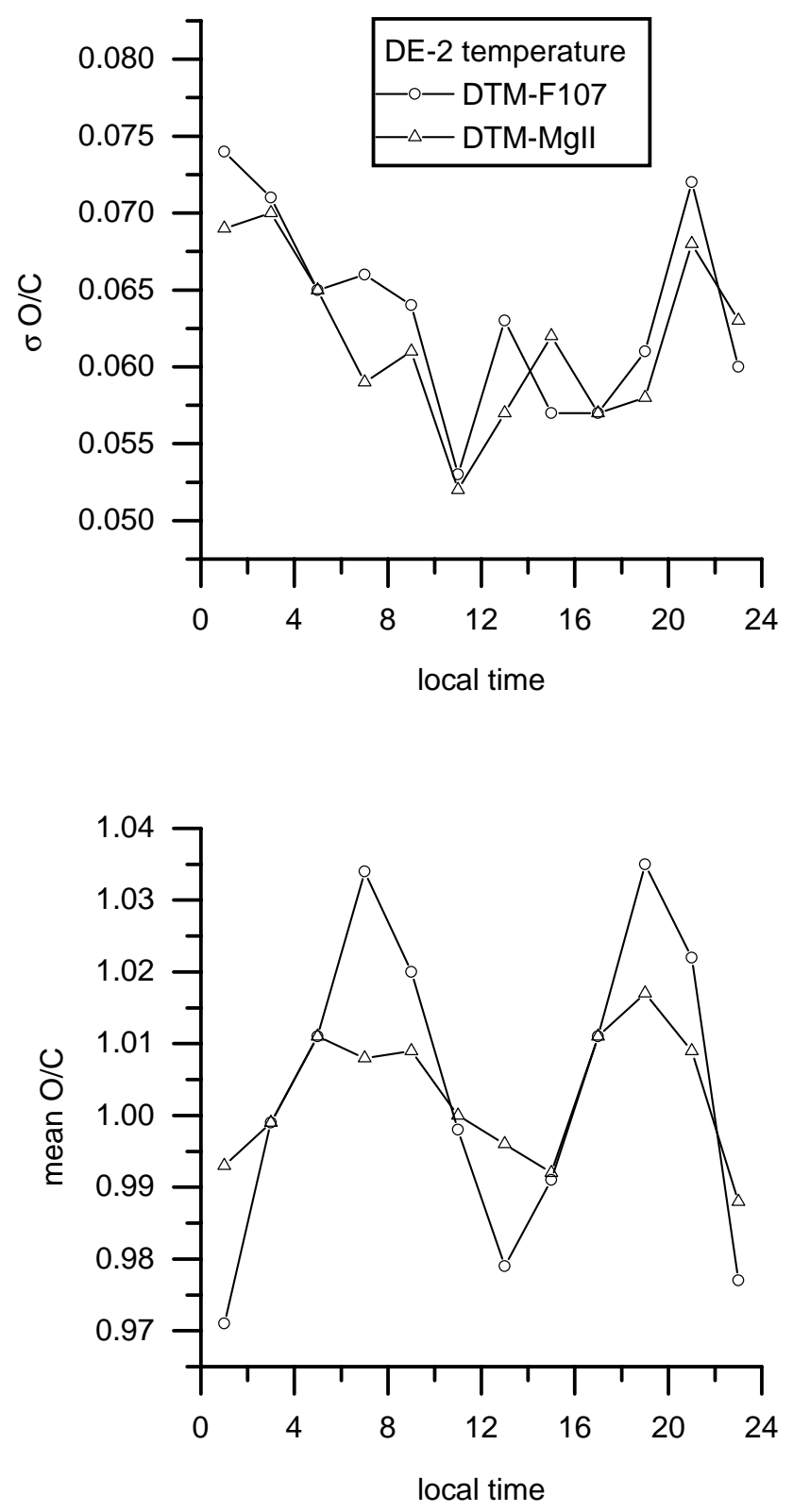

Fig. 5. The mean and scatter of the DE-2 temperature residuals presented in local time bins.

column of Table 1 . The global mean of the residuals is $0.5 \%$ biased, and the scatter of 0.062 is $6 \%$ smaller than that of the two other models. Fig. 5 presents the temperature residuals binned as a function of local time, which shows (bottom figure) biases of less than $1.5 \%$ for DTM-Mg II, while for DTM-F10.7, they are up to $3.5 \%$. The relative noise in the data is smallest around noon (top figure), which is when the signal to noise ratios are highest. Verification of the residuals after binning did not reveal systematic modelling errors.
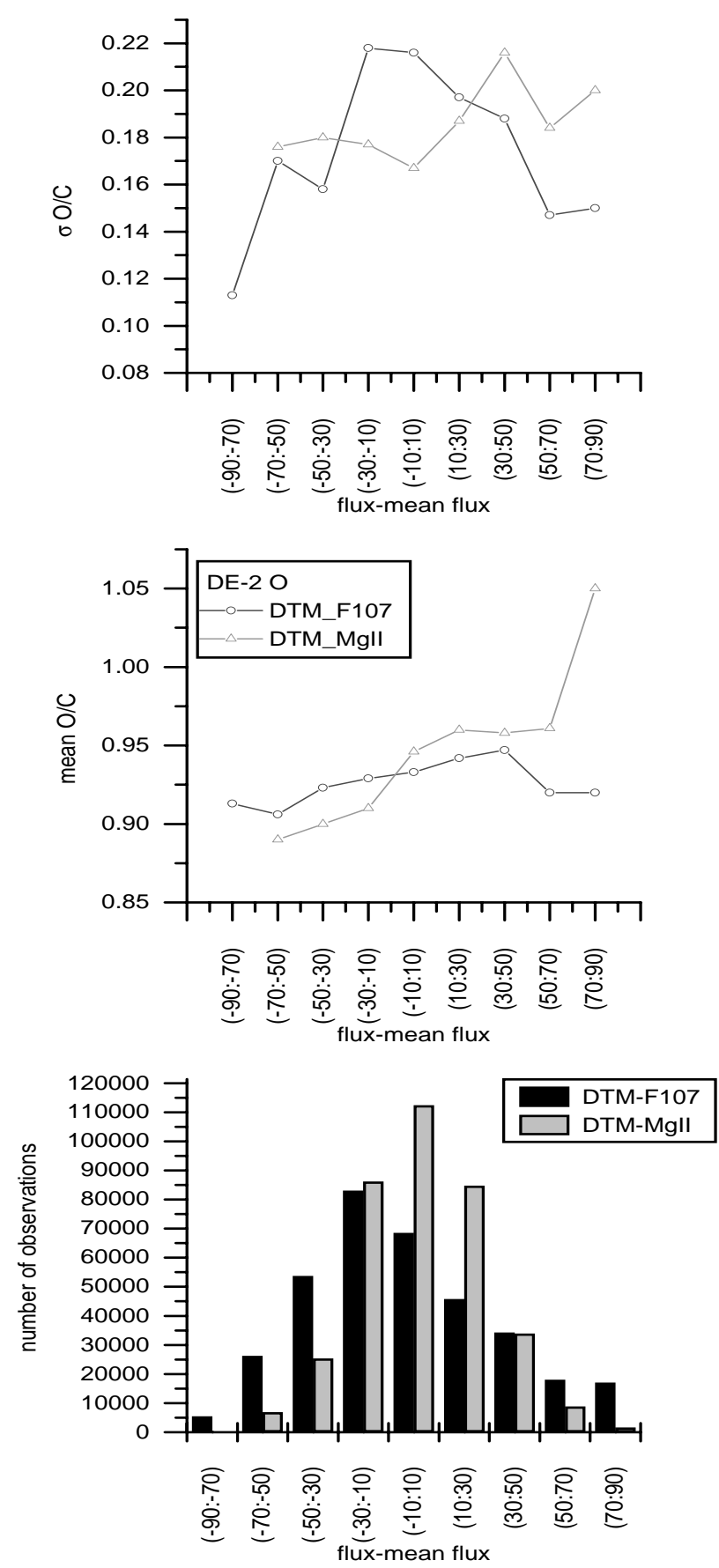

Fig. 6. The mean and scatter of the DE-2 oxygen residuals presented in F10.7 $-\overline{\mathrm{F}} 10.7$ bins, where $\overline{\mathrm{F}} 10.7$ represents the 27 -day mean of the solar radio flux or the rescaled $\mathrm{Mg}$ II index. The histogram at the bottom displays the distribution of F10.7 $-\overline{\mathrm{F}} 10.7$ at the observation epochs.

\subsection{The atomic oxygen modelling}

The atomic oxygen $(\mathrm{O})$ modelling is better with DTM-Mg II than with DTM-F10.7, as demonstrated by their respective 0.182 and 0.193 global scatter of the residuals in Table 1 . However, the biases are comparable, as expected, due to the 
Table 1. The modelling performance with respect to the DE-2 dataset. The number of residuals is given on the first line, followed by their mean and scatter on the second line.

\begin{tabular}{ccccc}
\hline & $\mathrm{He}$ & $\mathrm{O}$ & $\mathrm{N}_{2}$ & $\mathrm{~T}$ \\
\hline DTM-F107 & 513795 & 359729 & 226774 & 261976 \\
& $0.893 / 0.189$ & $0.930 / 0.193$ & $0.640 / 0.194$ & $1.009 / 0.067$ \\
\hline DTM-Mg II & 513635 & 359873 & 227022 & 261988 \\
& $0.933 / 0.195$ & $0.937 / 0.182$ & $0.627 / 0.188$ & $1.005 / 0.062$ \\
\hline
\end{tabular}

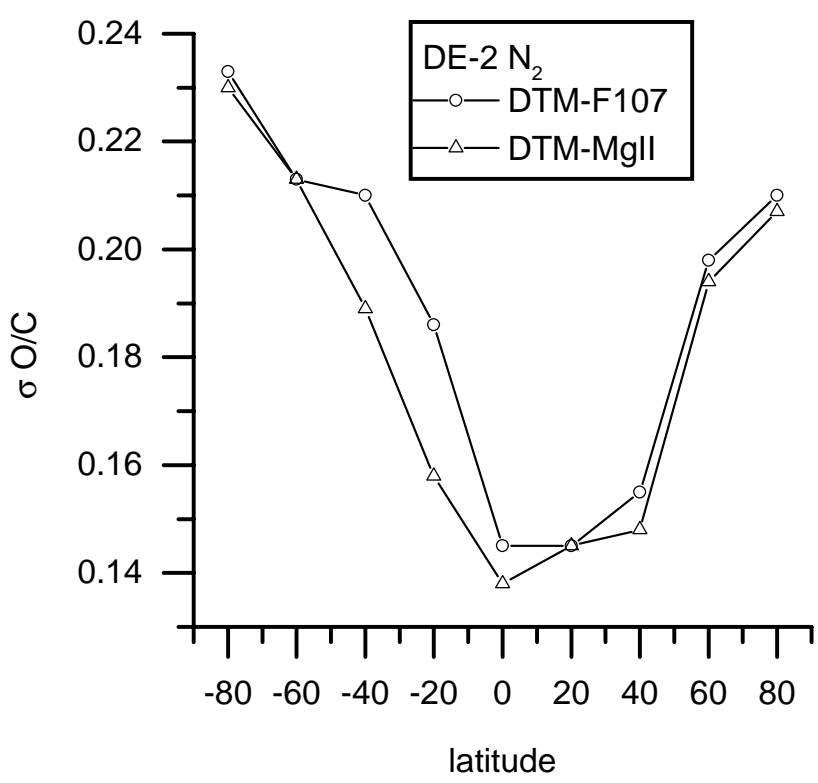

Fig. 7. The scatter of the DE-2 molecular nitrogen residuals presented in latitude bins.

use of the same scaling factor. Figure 6 presents the mean (middle) and RMS (top) of the residuals per (F10.7 $-\overline{\mathrm{F}} 10.7)$ bin for the test models. The histogram of the ( $\overline{\mathrm{F}} 10.7-\mathrm{F} 10.7)$ is shifted towards negative values for the 7/1981-1/1983 epoch, with most residuals in the $-30<\mathrm{F} 10.7-\overline{\mathrm{F}} 10.7<$ -10 bin, as shown in Fig. 6 (bottom). This is not the case for the $\mathrm{Mg}$ II index, whose distribution of differences is centred around 0 . The mean of the residuals per bin, on the other hand, is steadily increasing for both models (Fig. 6, middle). The curves displaying the means of the residuals are close, since, for both test models, the same DE-2 scaling factor was used, except for the poorly contributing bins (Fig. 6, bottom). The RMS of the residuals in the $(-30,30)$ interval, where $70 \%$ of the data is present, decreases from 0.22 to 0.17 (top figure).

Through further analysis (not shown) of the DTM-Mg II oxygen residuals, we learned that their smallest RMS is in the 200-400 km altitude range (where oxygen is a major constituent), approximately 0.13 , steadily increasing to 0.30 in the $600-700 \mathrm{~km}$ altitude range. A similar behaviour is seen in the DTM-F107 residuals, whose RMS' are approximately

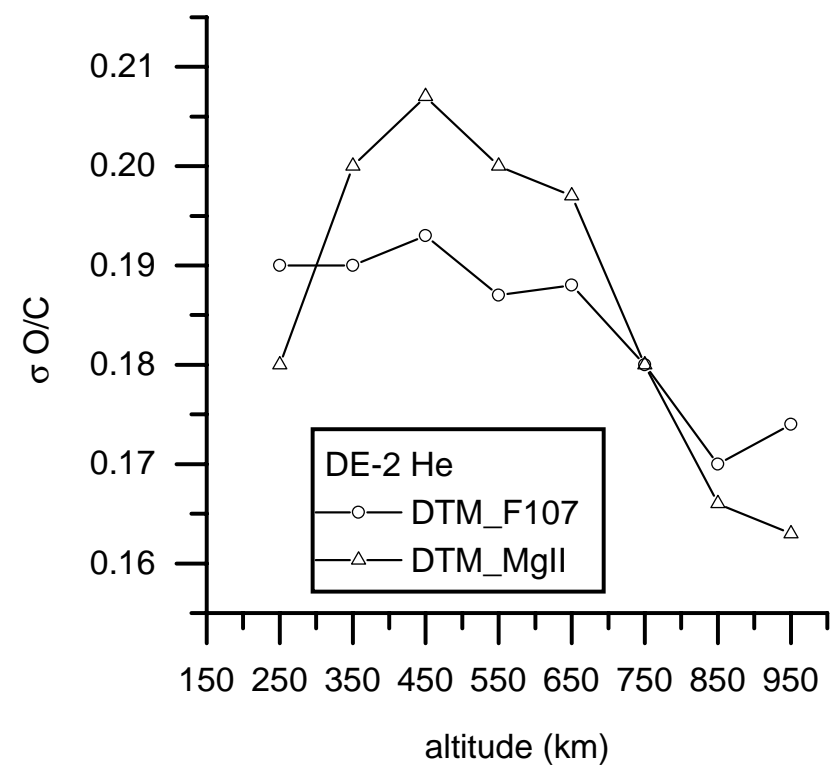

Fig. 8. The scatter of the DE-2 helium residuals presented in altitude bins.

$5 \%$ larger in the $200-400 \mathrm{~km}$ altitude range. The DTM-Mg II residuals display a typical latitudinal signature: higher values at the poles than at the equator. This behaviour is due to the auroral activity which induces short term perturbations of the neutral atmosphere, whose description, using the 3-hourly $K p$ indices, lacks temporal resolution. We have verified the modelling as a function of local time by binning the residuals per 2 hours. This showed that both test models have the same large step of approximately $12 \%$ between the 20-22 and 22$24 \mathrm{hr}$ local time-binned residuals. This is probably due to the data distribution.

It is important to note that (i) the RMS of the residuals per $\overline{\mathrm{F}} 10.7$ bin is the smallest for the highest solar activity bin (220-250 sfu), and (ii) the RMS of the 130-160 sfu bin is $18 \%$ smaller with DTM-Mg II than with DTM-F10.7. This is probably due to the weaker 27-day cycle of F10.7, which flattens out at solar minimum compared to Mg II (and thus, presumably the true EUV flux). Indeed, between 1985 and 1987 (minimum of solar cycle 22), the peak amplitude of the rescaled $\mathrm{Mg}$ II index was larger than F10.7 in $75 \%$ of the cases. The DTM-Mg II residuals binned as a function of 
$K p$ presented the most stable mean, up to $K p=6$, and the smallest RMS for $K p \leq 3$.

\subsection{The molecular nitrogen modelling}

The $\mathrm{N}_{2}$ DTM-Mg II modelling precision (RMS: 0.188) has improved 3\% with respect to DTM-F107 (0.194), as presented in Table 1. Verification of the latitude modelling by binning the residuals did not reveal the latitudinal signature as described in Sect. 3.2, but a mean per bin between 0.60 and 0.65 . However, Fig. 7 shows that this signature around the equator is present in the standard deviations of the binned residuals of the test models, i.e minimum at the equator due to the smallest perturbations caused by auroral activity.

Further analysis of the $\mathrm{N}_{2}$ residuals revealed that at altitudes below approximately $240 \mathrm{~km}$, where $\mathrm{N}_{2}$ becomes the major constituent, the $\mathrm{Mg}$ II model gained $10 \%$ in precision with respect to DTM-F107. Furthermore, the residuals binned per $\overline{\mathrm{F}} 10.7$ showed the smallest RMS with DTM-Mg II for the 220-250 sfu bin, 9\% better than DTM-F10.7. It was however, $16 \%$ less precise for a flux from 130 to $160 \mathrm{sfu}$.

\subsection{The helium modelling}

On short time scales, the helium modelling has slightly deteriorated using the Mg II index, as indicated by an increase of the scatter (0.195), as shown in Table 1. Most helium observations are in the $200-500 \mathrm{~km}$ altitude range. However, helium is a light element, and therefore, becomes the major constituent at altitudes exceeding 700 to $1000 \mathrm{~km}$, depending on solar activity. Figure 8 displays the residuals per altitude bin, showing the 3 to $6 \%$ smaller RMS with DTM-Mg II than with DTM-F10.7, in the 800 to $1000 \mathrm{~km}$ altitude range. The higher precision is probably due to the temperature modelling, which is best at high altitude because it is no longer affected by uncertainties in the modelling of its gradient.

Binning of the residuals as a function of the latitude presents means ranging from approximately 0.97 at the poles to 0.87 at the equator for DTM-Mg II, to be compared to 0.933 (Table 1). This variation is twice as large as with DTMF10.7. On the other hand, binning of the residuals as a function of local time uncovered a semidiurnal variation in the residuals, which is two times larger for DTM-F10.7 (mean per bin ranging from 0.83 to 0.97 ) than for DTM-Mg II.

The mean per $\overline{\mathrm{F}} 10.7$ bin ranges from $0.92-0.96$ with DTM$\mathrm{Mg}$ II, while the corresponding interval for DTM-F10.7 is 0.86-0.97, indicating that it does not represent changes in density as a function of mean flux correctly.

\subsection{The total density modelling}

An external validation of the model accuracies may be obtained by precise orbit computation (Marcos et al., 1993). By numerically integrating a satellite trajectory, with an accurate initial state vector, and computing residuals (observed satellite position minus computed satellite position), a direct orbit accuracy assessment is obtained. This is only possible in
Table 2. Comparison between the observed and computed position of GFZ-1 and Starlette during low (LF) and high (HF) solar activity. The mean RMS of the SLR residuals is given, in meters.

\begin{tabular}{ccc}
\hline & DTM-MgII & DTM-F107 \\
\hline GFZ-1 (LF) & 946 & 1193 \\
Starlette (LF) & 5 & 11 \\
Starlette (HF) & 9 & 16 \\
\hline
\end{tabular}

case of accurate and sufficiently dense tracking of the satellite. In this study, the Satellite Laser Ranging (SLR) system was used. A second and equally important condition is the employment of the most accurate force models in the orbit computation. Otherwise, errors not due to the atmospheric drag, but due to the Earth's gravity and solar radiation pressure modelling, in particular, may also be absorbed by the estimation of drag coefficients.

The geodetic satellites GFZ-1 and Starlette have been chosen for this evaluation. They are equipped with corner-cube reflectors to optimise the SLR tracking. Both satellites are in an approximately $50^{\circ}$ inclined orbit. GFZ- 1 was in a circular orbit at $380 \mathrm{~km}$ altitude initially and has decayed in June 1999. Starlette's orbit is eccentric $(e=0.02)$ with a perigee height of $800 \mathrm{~km}$ and an apogee height of $1100 \mathrm{~km}$. GFZ-1 was the only target in low altitude Earth orbit. SLR data are available from June 1995 until October 1997, i.e. during low solar activity only (the solar activity was at a minimum in 1996). Starlette was launched in 1975, allowing orbit computation under high and low solar activity conditions.

Under low solar activity, the major atmospheric constituents causing the drag force on GFZ-1 are $\mathrm{O}$ and $\mathrm{N}_{2}$. At $800 \mathrm{~km}$ (Starlette) and under similar conditions, helium is the unique major constituent. Under high solar activity conditions, the major constituents are $\mathrm{O}$ and $\mathrm{He}$ at that altitude.

The results of the GFZ-1 precise orbit tests are given in Table 2. The RMS of the fit relates to the precision of the restituted satellite motion after comparison to 4 days of SLR measurements, in which time GFZ-1 made approximately 64 orbital revolutions. This has been done for 5 different epochs, distributed over one year, resulting in the displayed average value of the RMS of the residuals. The GFZ-1 orbit computation benefits from the use of the Mg II index, demonstrated by the smaller RMS of the SLR residuals (946 m RMS on the average, compared to $1193 \mathrm{~m}$ in Table 2). These results corroborate with Table 1, which showed a more precise $\mathrm{O}$, $\mathrm{N}_{2}$ and temperature modelling using $\mathrm{Mg}$ II rather than using F10.7.

The results obtained with Starlette are also given in Table 2. The values are smaller than with GFZ-1 (approximately 10 vs. $1000 \mathrm{~m}$ ) because the density model error has a two orders of magnitude smaller impact on the orbit prediction due to the smaller absolute density at Starlette's altitude. The average RMS of the residuals represents the mean precision of the restituted orbital motion obtained by comparison to SLR 
data using 5 epochs of 8 days, computed during low solar activity (LF). A similar computation has been done during high solar activity (HF). The Starlette orbits have the smallest RMS of the SLR residuals with DTM-Mg II during low solar activity (5 m). Using the DTM- F107 model yields SLR residual RMS values that are approximately $40 \%$ larger than the corresponding DTM-Mg II values.

For all cases, under low and high solar activities, using DTM-Mg II gives better orbit predictions.

\section{Discussion}

The Mg II index and F10.7 present a high correlation over an entire solar cycle (0.99), but not on shorter time scales, in particular, the one linked to the solar rotation. The above is reflected in the modelling when the Mg II index is used instead of F10.7. The Mg II index's capability for thermosphere modelling has been evaluated using only DE-2 data, spanning 1.5 years during high to moderate solar activity. This period of time is sufficient to produce the test models, but the density variation as a function of solar activity and season cannot be accurately estimated. At least an entire solar cycle of data is required to elaborate a homogeneous and representative new DTM model. However, the results obtained with DTM-Mg II are better than with DTMF107. DTM-F107 suffers more from the short time span of the data than DTM-Mg II. The modelling results are better with $\mathrm{Mg}$ II, and even more so under high solar activity, than with F10.7, as was shown in Table 2. Not surprisingly, it is just under those conditions that the difference between these proxies becomes large, as was shown in Fig. 4.

The CHAMP mission (DLR, CNES, NASA) was launched in July 2000, and will provide accurate total density data thanks to its accelerometer, and GPS and SLR tracking systems. Due to the natural decay of its nearly polar orbit, total densities from $470 \mathrm{~km}$ down to $200 \mathrm{~km}$ will be measured over a period of approximately 5 years, including the entire declining phase of the solar cycle 23. When these data will be combined with $\mathrm{Mg}$ II observations, the density dataset will be 6.5 years, compared to the 1.5 years today. The required longer time span of the helium data with $\mathrm{Mg}$ II observations may be acquired using the satellite drag total density technique, and the geodetic satellites Starlette and Stella (at 800 $\mathrm{km}$ altitude and an inclination of $98^{\circ}$ ), for example.

\section{Conclusion}

Comparison of the test models DTM-Mg II and DTM-F107 demonstrated the superior representativity of the Mg II index with respect to upper atmosphere heating processes, in particular, under high solar activity conditions. The smaller standard deviations of the DE- 2 residuals at altitudes where they represent the major constituents show a 3 to $8 \%$ higher precision. The orbit tests with DTM-Mg II present 20-40\% smaller RMS of the SLR residuals than with DTM-F107. The fact that the density model based upon $\mathrm{Mg}$ II is more accurate than that based upon F10.7 under high solar activity, where these proxies differ most, demonstrates the higher representativity of the $\mathrm{Mg}$ II index of upper atmosphere heating processes. The lack of density data under low solar activity introduced a bias in the test models, and a larger and more representative density dataset is required to elaborate a homogeneous DTM model based upon the Mg II index.

The Mg II index as a UV-proxy is well acknowledged. By this study, it appears also usable in the EUV domain. It would be important to study, from a solar and upper atmosphere physics point of view, the basic reason of the success of $\mathrm{Mg}$ II in density (heating) modelling of the Earth's atmosphere.

Acknowledgements. This study benefitted from the support of the Centre National d'Etudes Spatiales. The authors thank R. Cebula for kindly providing us with the $\mathrm{Mg}$ II data. We also wish to thank G. Kockarts and the second referee for helpful comments. The model algorithm and coefficients are available upon request (sean. bruinsma@cnes.fr or gerard.thuillier@aerov.jussieu.fr).

Topical Editor D. Murtagh thanks G. J. Kockarts and R. Viereck for their help in evaluating this paper.

\section{References}

Avrett, E. H., Temporal variations of near-UV, visible, and infrared spectral irradiance from a theoretical viewpoint, Proceedings of the Workshop on the Solar Electromagnetic Radiation Study for Solar Cycle 22 (Ed. R.F. Donnelly), National Technical Information Service, Springfield, VA, 20-42, 1992.

Barlier, F., Berger, C., Falin, J.L., Kockarts, G., and Thuillier, G., A thermospheric model based on satellite drag data, Ann. Geophysicae, 34, 9-24, 1978.

Bates, D. R., Some problems concerning the terrestrial atmosphere above about the 100-km level, Proc. R. Soc. A., 253, 451-462, 1959.

Berger, C., Biancale, R., Ill, M., and Barlier, F., Improvement of the empirical thermospheric model DTM: DTM94-comparative review on various temporal variations and prospects in space geodesy applications, J. Geodesy, 72, 161-178, 1998.

Carignan, G. R., Block, B. P., Maurer, J.C., Hedin, A. E., Reber, C. A., and Spencer, N. W., The neutral mass spectrometer on Dynamics Explorer, Space Sci. Instrum., 5, 429-441, 1981.

Cebula, R. P. and DeLand, M. T., Comparisons of the NOAA-11 SBUV/2, UARS SOLSTICE and UARS SUSIM Mg II solar activity proxy indices, Solar Phys., 177, 117-132, 1998.

Cebula, R. P., DeLand, M. T., and Hilsenrath, E., NOAA-11 Solar Backscattered Ultraviolet, model 2 (SBUV/2) instrument solar spectral irradiance measurements in 1989-1994 1. Observations and long-term calibration, J. Geophys. Res., 103, 16235-16249, 1998.

DeLand, M. T. and Cebula, R. P., Composite Mg II solar activity index for solar cycles 21 and 22, J. Geophys. Res., 98, 1280912823, 1993.

Donnelly, R. F., White, O. R., and Livingston, W. C., The solar Ca II index and the Mg II core-to-wing ratio, Solar Phys., 152, 69-76, 1994.

Donnelly, R. F. (Ed.), Proceedings of the Workshop on the Solar Electromagnetic Radiation Study for Solar Cycle 22, National Technical Information Service, Springfield, VA, 275-285, 1992. Donnelly, R. F. and Puga, C., Thirteen-day periodicity and the 
center-to-limb dependence of UV, EUV, and X-ray emission of solar activity, Solar Phys., 130, 369-390, 1990.

Heath, D. F. and Schlesinger, B. M., The Mg $280 \mathrm{~nm}$ doublet as a monitor of changes in solar ultraviolet irradiance, J. Geophys. Res., 91, 8672-8682, 1986.

Hedin, A. E., Correlations between thermospheric density and temperature, solar EUV flux, and 10.7-cm flux variations, J. Geophys. Res., 89, 9828-9834, 1984.

Hedin, A. E., MSIS-86 thermospheric model, J. Geophys. Res., 92, 4649-4662, 1987.

Jacchia, L. G., Revised static models of the thermosphere and exosphere with empirical temperature profiles, Special Report 332, Smith. Astrophys. Obs., 1-113, 1971.

Lean, J. L., White, O. R., Livingston, W. C., Donnelly, R. F., and Skumanich, A., A three-component model of the variability of the solar ultraviolet flux: 145-200 nm, J. Geophys. Res., 87, 10307-10317, 1982.

Lean, J. L., Vanhoosier, M., Brueckner, G., and Prinz, D., SUSIM/UARS observations of the 120 to $300 \mathrm{~nm}$ flux variations during the maximum of the solar cycle: inference for the 11-year solar cycle, Geophys. Res. Lett., 19, 2203-2206. 1992.

Marcos, F. A., Baker, C. R., Bass, J. N., Killeen, T. L., and Roble, R. G., Satellite drag models: current status and prospects, paper AAS 93-621, AAS/AIAA Astrodynamics Specialist Conference, Victoria, B.C., Canada, 1993.

Neupert, W. M., Spatial distribution of sources of EUV emission: correlation of EUV and microwave emission with Ca II K plage, Solar Phys., 177, 181-190, 1998.

Nier, A. O., Potter, W.E., Hickman, D. R., and Mauersberger,
K., The open-source neutral mass spectrometer on Atmosphere Explorer-C, -D, -E, Radio Sci., 8, 271-276, 1973.

Oliver, W. L., Incoherent scatter radar studies of the daytime thermosphere, Ann.Geophysicae, 35, 121-135, 1979.

Reigber, Ch., Bock, R., Förste, Ch., Grunwaldt, L., Jakowski, N., Lühr, H., Schwintzer, P., and Tilgner, C., CHAMP Phase B Executive Summary, Scientific Technical Report STR96/13, GeoForschungsZentrum Potsdam, Germany, 1996.

Spencer, N. W., Niemann, H. B., and Carignan, G. R., The neutralatmosphere temperature instrument, Radio Sci., 8, 284-296, 1973.

Spencer, N. W., Wharton, L. E., Niemann, H. B., Hedin, A. E., Carignan, G. R., and Maurer, J. C., The Dynamics Explorer wind and temperature spectrometer, Space Sci. Instrum., 5, 417-428, 1981.

Thuillier, G., Falin, J. L., and Barlier, F., Global experimental model of the exospheric temperature using optical and incoherent scatter measurements, J. Atmos. Terr. Phys., 39, 1195-1202, 1977.

Viereck R. A., and Puga, L. C., The NOAA Mg II core-to-wing solar index: Construction of a 20-year time series of chromospheric variability from multiple satellites, J. Geophys. Res., 104, 999510005, 1999.

Villain, J. P., Traitement des données brutes de l'accéléromètre CACTUS. Etude des perturbations de moyenne échelle de la densité thermosphérique, Ann. Geophysicae, 36, 41-49, 1980.

White, O. R., Livingston, W. C., Kiel, S. L., Proceedings of the Workshop on the Solar Electromagnetic Radiation Study for Solar Cycle 22, National Technical Information Service, Springfield, VA, 160-165, 1992. 